\title{
Can lattice data for two heavy-light mesons be understood in terms of simply two-quark potentials?
}

\author{
A.M. Green*, J. Koponen ${ }^{a}$ and P. Pennanen ${ }^{\mathrm{b}}$ \\ a Department of Physics and Helsinki Institute of Physics, P.O. Box 9, FIN-00014 University of \\ Helsinki, Finland (email: anthony.green@helsinki.fi, jmkopone@rock.helsinki.fi) \\ bordita, Blegdamsvej 17, 2100 Copenhagen $\varnothing$, Denmark (email: petrus@hip.fi)
}

By comparing lattice data for the two heavy-light meson system $\left(Q^{2} \bar{q}^{2}\right)$ with a standard many-body approach employing only interquark potentials, it is shown that the use of unmodified two-quark potentials leads to a gross overestimate of the binding energy.

\section{INTRODUCTION}

In this conference the emphasis is on the study of single hadrons, with virtually nothing being said about systems involving more than three quarks. This is unfortunate, since it avoids much of particle physics and all of nuclear physics. It is, therefore, desirable to attempt to bridge this gap. Since the ability to perform accurate lattice simulations for systems containing many quarks is severely limited - with four quarks being the current maximum - the challenge is to see how (if) standard many-body techniques can be developed for the new situation of interacting quarks and gluons. Here we immediately meet a cultural difference. For particle physicists, the use of simply two-quark potentials in multiquark problems is not a discussion point, since they know (believe) that it is not possible. In spite of this, there are many-body physicists, who still believe (hope) that this is not so.

Over the last few years attempts have been made to clarify this situation by comparing the exact energies of four-quark systems - as calculated on a lattice - with standard many-body models using only two-quark potentials [1]. The four-quark system was studied for two reasons: i) Accurate lattice simulations are still possible. ii) It is the first step in the description of interacting hadrons, since the system can be partitioned

\footnotetext{
*Presented at Lattice '99 in Pisa, Italy.
}

into two colour singlets. The outcome from those pilot comparisons was that - "beyond all reasonable doubt" - the resulting binding energies are grossly overestimated by the models [Figure 1] and that a four-quark form factor is necessary. However, this conclusion was based on lattice data for four static quarks in quenched $\mathrm{SU}(2)$. In the present work [2] most of these approximations have been removed by using $\mathrm{SU}(3)$ with two heavy-light mesons i.e. $Q^{2} \bar{q}^{2}$ compared with the earlier $Q^{2} \bar{Q}^{2}$. Furthermore, the gauge field is now treated in the unquenched approximation. But this latter improvement only has a small effect. The corresponding many-body model now needs some additional assumptions before a comparison with the lattice data is possible. Perhaps the most serious is the use of a non-relativistic kinetic energy, even though the light quark mass is approximately that of the strange quark. However, in the binding energy there is a considerable cancellation between the four- and two-quark kinetic energies, which then removes much of the kinetic energy effect from the binding energy. Also the model is developed only for the spin-independent contribution to the binding energy. This requires an averaging of the lattice data. Both of these assumptions are not thought to qualitatively effect the outcome. The main defect of the calculation is that, at present, comparison can only be made for small distances between the two static quarks - the region where lattice data could suffer from 


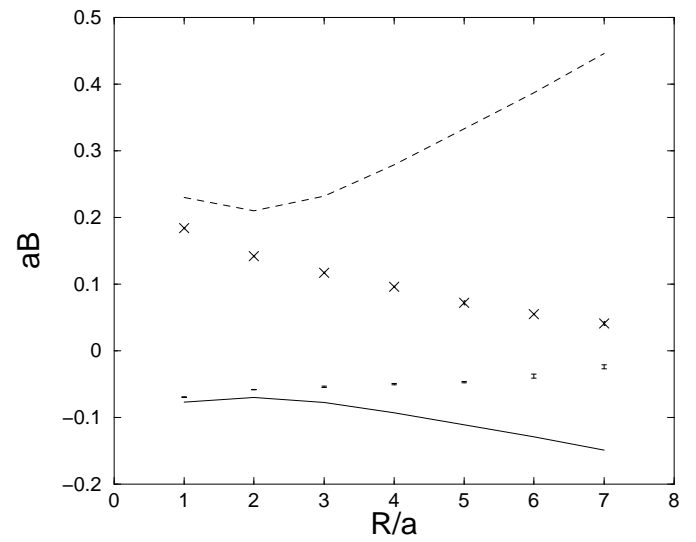

Figure 1. The case of four static quarks $\left(Q^{4}\right)$ in $\mathrm{SU}(2)$ placed at the corners of squares of side $R / a$, where $a \approx 0.12 \mathrm{fm}$. This shows the binding energy in lattice units $(a B)$ as a function of $R / a$. The lattice results are for $\beta=2.4$ on a $16^{3} \times 32$ lattice with the dots (crosses) showing the ground (excited) state energies - with error bars. The two curves are for the model resulting in Eq. 6 (with $k_{f}=0$ ) and correspond to the ground (solid line) and excited (dashed line) states. This figure is similar to the one in [1]

a lack of rotational invariance. However, in spite of these shortcomings, the result is that the use of simply two-quark potentials again overestimates the lattice binding by upto a factor of three a result that is not expected to be qualitatively changed by other models without multi-quark interactions [Figure 2-solid line]. Inclusion of a four-quark interaction term can then remove the discrepancy [Figure 2 - dashed line].

The reason why the "data-model" comparison is good for small $R / a$ in Figure 1 and not in Figure 2 is simply because the $Q^{2} \bar{q}^{2}$ energy - even for small $R$ - is dominated by potential energy contributions, where the $Q \bar{q}$ and $\bar{q} \bar{q}$ separations are $\approx 3 a$. As seen in Figure 1, at such separations the model already becomes too attractive in the absence of the form factor $f$.

\section{MODEL}

In Refs. 11,3, 国 a model was developed for understanding the lattice energies of four static

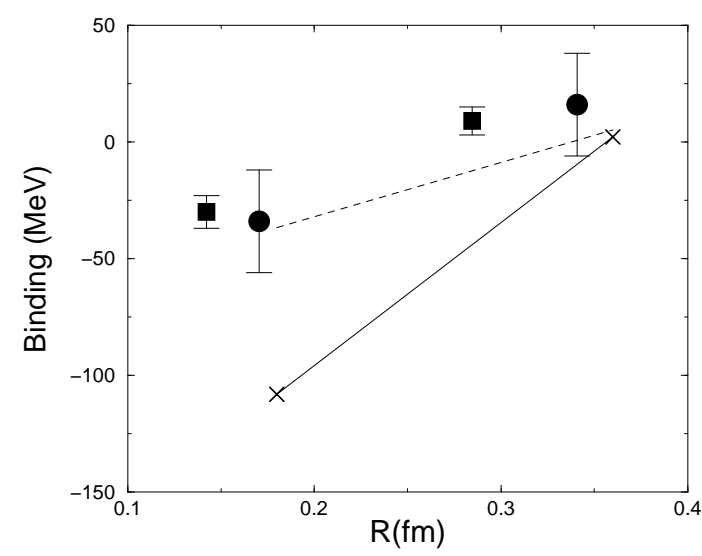

Figure 2. Comparison between the spin independent part $\left(V_{0}\right)$ of the $Q^{2} \bar{q}^{2}$ binding energies calculated on a lattice [2] (solid circles - quenched approximation with $a=0.170 \mathrm{fm}$ )/ [5] (solid squares - with dynamical fermions and $a=0.142$ $\mathrm{fm})$ and the model in the weak coupling limit $\left(k_{f}=0\right)$ - solid line. The dashed line shows the model prediction for $k_{f}=0.25$. These lines are to guide the eye. The dynamical fermion data is not used in any fit. It is simply included to show that it is qualitatively consistent with the quenched data but with considerably smaller error bars.

quarks

$Q\left(\mathbf{r}_{\mathbf{1}}\right) Q\left(\mathbf{r}_{\mathbf{2}}\right) \bar{Q}\left(\mathbf{r}_{\mathbf{3}}\right) \bar{Q}\left(\mathbf{r}_{\mathbf{4}}\right)$ in terms of two-quark potentials. This model, in its simpliest form, was constructed in terms of the two basis states that can be made by partitioning the four quarks into two color singlets - namely -

$$
\begin{aligned}
A & =[Q(1) \bar{Q}(3)][Q(2) \bar{Q}(4)] \\
B & =[Q(1) \bar{Q}(4)][Q(2) \bar{Q}(3)],
\end{aligned}
$$

where $[\ldots]$ denotes a color singlet. These two states are not orthogonal and have a normalisation matrix of the form.

$\mathbf{N}(\mathbf{f})=\left(\begin{array}{ll}1 & \frac{1}{3} f \\ \frac{1}{3} f & 1\end{array}\right)$.

In the extreme weak coupling limit the parameter $f=1$ and in the strong coupling limit $f=0$. However, for intermediate situations it is parametrised as

$f\left(\mathbf{r}_{\mathbf{1}}, \mathbf{r}_{\mathbf{2}}, \mathbf{r}_{\mathbf{3}}, \mathbf{r}_{\mathbf{4}}\right)=\exp \left[-b_{s} k_{f} S\left(\mathbf{r}_{\mathbf{1}}, \mathbf{r}_{\mathbf{2}}, \mathbf{r}_{\mathbf{3}}, \mathbf{r}_{\mathbf{4}}\right)\right],(3)$ 
where $b_{s}$ is the string energy, $S\left(\mathbf{r}_{1}, \mathbf{r}_{2}, \mathbf{r}_{3}, \mathbf{r}_{\mathbf{4}}\right)$ is an area defined by the positions of the quarks and $k_{f}$ is a free parameter. A single value for $k_{f}$ was then capable of giving a reasonable understanding for 100 pieces of data - the ground and first excited states of configurations from six different fourquark geometries calculated on a $16^{3} \times 32$ lattice. In this model the interaction between the quarks is expressed as a potential matrix of the form

$\mathbf{V}(\mathbf{f})=\left(\begin{array}{ll}v(13)+v(24) & f V_{A B} \\ f V_{A B} & v(14)+v(23)\end{array}\right)$,

where $V_{A B}$ has the form expected in the weak coupling limit with the one-gluon-exchangepotential

$V=-\frac{1}{3} \sum_{i \leq j} \lambda_{i} \lambda_{j} v(i j)$ and $v(i j)=-\frac{e}{r_{i j}}$.

Away from the weak coupling limit, $f$ is no longer unity and in addition $v(i j)$ is taken to be the full two quark potential. The energy of the four static quarks - a function of the $\mathbf{r}_{\mathbf{i}}-$ is then given by diagonalising

$\left|\mathbf{V}\left(k_{f}\right)-E\left(4, \mathbf{r}_{\mathbf{i}}, k_{f}\right) \mathbf{N}\left(k_{f}\right)\right| \psi=0$.

This model is easy to generalise to $Q^{2} \bar{q}^{2}$ by integrating over the positions of the two light quarks using a suitable variational wavefunction [3]. This results in the need to diagonalise

$$
\left|\mathbf{K}\left(k_{f}\right)+\mathbf{V}\left(k_{f}\right)-E\left(4, R, k_{f}\right) \mathbf{N}\left(k_{f}\right)\right| \psi=0,
$$

where $\mathbf{K}\left(R, k_{f}\right)$ is the kinetic energy and $R$ is the distance between the two static quarks. In order to carry out the radial integrals in $\mathbf{K}\left(R, k_{f}\right), \mathbf{V}\left(R, k_{f}\right)$ and $\mathbf{N}\left(R, k_{f}\right)$, the function $f$ in Eq. 3 was now reduced to the more symmetric form

$f=\exp \left[-k_{f} b_{s} \sum_{i \leq j} r_{i j}^{2}\right]$.

Again $k_{f}$ is a free parameter, which should be adjusted to fit the four-quark lattice energies. However, now only the lattice data for $R=a$ is sufficiently accurate to extract a value of $k_{f}$ - see Figure 2.
The present model is now being developed to include:

1) a semi-relativistic approximation, in which for the two light quarks the kinetic energy operator in momentum space is simply replaced by $\sqrt{p_{i}^{2}+m_{q}^{2}}-m_{q}$.

2) a spin dependence in the potential between the two light quarks.

This model, although very simple, contains the same basic assumptions made in the more elaborate many-body models that incorporate kinetic energy e.g. the Resonating Group Method [6]. It is, therefore, reasonable that this simplified model can to some extent check the validity of its more elaborate counterparts.

\section{CONCLUSION}

The comparison between lattice data and a model for the $Q^{2} \bar{q}^{2}$ system supports (confirms) the earlier result - with four static quarks in quenched $\mathrm{SU}(2)$ - that four-quark energies cannot be described simply in terms of two-quark potentials and that attempts to do so could lead to a large overestimate of the binding energy. One way of overcoming this problem is to include into the model a four-quark form factor - as seen in Figure 2.

\section{REFERENCES}

1. A.M. Green, C. Michael and J. Paton, Nucl.Phys. A554, 701 (1993).

2. C. Michael and P. Pennanen, Phys.Rev. D 60, 54012 (1999), hep-lat/9901007.

3. A.M. Green, J. Koponen and P. Pennanen, hep-ph/9902249.

4. A.M. Green and P. Pennanen, Phys. Rev. C 57, 3384 (1998), hep-lat/9804003.

5. UKQCD Collaboration: C. Michael and P. Pennanen, work in progress.

6. M. Oka and Yazaki, "Quarks and Nuclei", Int. Review of Nuclear Physics - Vol. 1 (1984) p.489, eds. T.T.S.Kuo and E.Osnes (World Scientific Publishing Co. Singapore) 